\title{
Loss of cytokeratin 10 indicates malignant transformation in actinic cheilitis
}

\author{
Natália Galvão Garcia ${ }^{1}$ - Denise Tostes Oliveira ${ }^{1}$. José Roberto Pereira Lauris ${ }^{2}$ \\ Maria Aparecida Custódio Domingues ${ }^{3}$ - Eliana Maria Minicucci ${ }^{3}$. \\ Cléverson Teixeira Soares ${ }^{4}$
}

Received: 24 January 2015 / Accepted: 31 July 2015 / Published online: 13 August 2015

(C) Springer-Verlag Berlin Heidelberg 2015

\begin{abstract}
Objectives The aim of this study was to investigate the relationship the expression of cytokeratins (CK10 and CK13) and the cell proliferation index determined by Ki-67 of lip squamous cell carcinoma and actinic cheilitis with different degrees of dysplasia.

Materials and methods Forty-five paraffin-embedded actinic cheilitis with and without dysplasia and 20 lip squamous cell carcinoma were analyzed by immunohistochemistry using anti-human anti-CK10, anti-CK13, and anti-Ki-67 antibodies. Results The majority of actinic cheilitis showed immunopositivity for CK10 and CK13 with decrease or loss of expression in dysplastic areas. In lip squamous cell carcinoma of the lip, heterogeneous expression of CK13 and immunonegativity for CK10 were observed. There was a statistically significant difference between CK10 expression in lip squamous cell carcinoma and in actinic cheilitis with or without dysplasia $(p<0.001)$. The cell proliferation index was higher in actinic cheilitis with dysplasia and lip squamous cell carcinoma
\end{abstract}

Natália Galvão Garcia natggalvao@hotmail.com

Denise Tostes Oliveira denisetostes@usp.br

1 Department of Stomatology, Area of Pathology, Bauru School of Dentistry, University of São Paulo, Al. Dr. Octávio Pinheiro Brisolla, 9-75, Bauru, São Paulo 170120-901, Brazil

2 Department of Community Dentistry, Bauru School of Dentistry, University of São Paulo, Bauru, São Paulo, Brazil

3 Department of Pathology, Botucatu School of Medicine, State University of São Paulo, Botucatu, São Paulo, Brazil

4 Department of Pathology, Lauro de Souza Lima Institute, Bauru, São Paulo, Brazil than in actinic cheilitis without epithelial dysplasia. A significant correlation was found between the intensity of the epithelial dysplasia and the cell proliferation index $(p<0.001)$.

Conclusion These results provide evidence that there is a downregulation of CK10 expression in dysplastic areas of patients with actinic cheilitis and in those with lip squamous cell carcinoma (LSCC) and that the index of cell proliferation, determined by $\mathrm{Ki}-67$, is directly correlated with the intensity of the epithelial dysplasia.

Clinical relevance Altogether, these results suggest that CK10 expression and the epithelial cell proliferation index can help to identify malignant transformation in the lip region.

Keywords Actinic cheilitis - Epithelial dysplasia - Squamous cell carcinoma of the lip · Cell proliferation index

\section{Introduction}

The presence of dysplastic areas in the epithelium of the oral mucosa in some lesions is frequently associated with progression of oral cancer [1]. However, the prognostic value of epithelial dysplasia in these potentially malignant lesions is difficult to determine [2]. Currently, no molecular marker has proved to be prognostically significant, and none have yet been evaluated in large prospective studies in these oral lesions $[1,2]$.

The diagnosis and intensity of oral epithelial dysplasia are based on a combination of architectural and cytological changes [3]. However, the microscopic evaluation of dysplasia remains subjective, and the graduation of its intensity has induced considerable inter and intra observer variations $[1,2,4,5]$.

In some cases of actinic cheilitis arising in the vermillion of the lower lip, a potentially malignant lesion induced by solar radiation, epithelial dysplasia is frequently presented $[6,7]$. Although there is no accurate information about the 
prevalence of malignant transformation of actinic cheilitis, the presence and intensity of epithelial dysplasia increases the chance of progression to lip cancer [8,9]. In tropical countries such as Brazil, there is a high incidence of actinic cheilitis and lip cancer [8]; therefore, the identification of microscopic epithelial dysplasia in these lesions is an important predictive factor in determining the risk of lip carcinoma [6-9].

It has been suggested that the cytokeratin 10 (CK10) and 13 (CK13) are markers of dysplastic epithelium, and they can be used to assess the malignant progression in premalignant lesions with dysplasia [10-17]. However, information about the immunohistochemistry pattern of these cytokeratins in actinic cheilitis are scarce.

This study was designed to investigate the immunoexpression of cytokeratin 10 and 13 and their association with epithelial cell proliferation index in tissues of patients presenting actinic cheilitis with or without dysplasia and in those with lip squamous cell carcinoma (LSCC), in order to contribute with the identification of biomarkers associated with malignant transformation in the lip.

\section{Materials and methods}

\section{Patients}

This study was based on the analysis of 65 patients who received surgical treatment for actinic cheilitis or LSCC at the Department of Otorhinolaryngology and Ophthalmology of Botucatu School of Medicine, São Paulo, Brazil. This study was approved by the Research Ethics Committee of the Botucatu School of Medicine, São Paulo State University, (UNESP), São Paulo, Brazil (Process Number 75 /2012).

The data of 45 patients presenting actinic cheilitis with and without epithelial dysplasia and 20 patients with lip squamous cell carcinoma were obtained from the Department of Pathology archives, and the formalin-fixed, paraffin-embedded tissue blocks were cut into 3- $\mu \mathrm{m}$ sections for hematoxylin and eosin (H\&E) staining and immunohistochemistry analysis of cytokeratins (CK10 and CK13) and anti-Ki- 67. The tumor sections were examined by three pathologists (NGG, DTO, MACD), and the histopathological dysplasia grade was established, based on epithelial changes determined by World Health Organization (3) including the loss of polarity of the basal cells, the hyperplasia of basal cells, the increased nuclear-cytoplasmic ratio, the drop-shaped rete ridges, acanthosis and atrophy, irregular epithelial stratification, cellular and nuclear pleomorphism, nuclear hyperchromatism, mitotic figures, and multinucleated cells. Lesions were graded as follows: actinic cheilitis without epithelial dysplasia $(26.6 \%)$ and actinic cheilitis showing mild dysplasia ( $24.4 \%$ of cases) or moderate dysplasia (31.0\% of cases) or severe dysplasia ( $18.0 \%$ of cases).

\section{Immunohistochemistry}

The sections of actinic cheilitis and LSCC were deparaffinized in xylene and hydrated using graded alcohol/water baths. Antigen retrieval was performed using $10 \mathrm{mM}$ citrate buffer, $\mathrm{pH} 6.0$, in a pressure cooker (Pascal S2800, Dako, Brazil) for $3 \mathrm{~min}$, and the endogenous peroxidase activity was blocked by incubation in $3 \% \mathrm{H}_{2} \mathrm{O}_{2}$ for $20 \mathrm{~min}$. Each actinic cheilitis or lip cancer section was incubated overnight at $4{ }^{\circ} \mathrm{C}$ with a primary monoclonal anticytokeratin-13 (Clone KS-1A3, Genetex-USA-1:100 dilution) or with a primary monoclonal anti-cytokeratin-10 - (Clone LHP1, Novocastra, UK-1:100 dilution) or with a primary monoclonal anti-Ki-67 (Clone MIB-1 M7240, Dako, North America-1:100 dilution.). Primary antibody dilutions were made in a phosphatebuffered saline (PBS)/bovine serum albumin (Sigma, Co., St Louis, MO, USA) solution to block nonspecific reactions. Then, the sections were incubated using the Advance HRP Link System (Dako North America, Inc.) for $30 \mathrm{~min}$ at $37^{\circ} \mathrm{C}$. The antibodies anti-CK13, anti-CK10, and anti-Ki-67 were detected using 3'3 diaminobenzidine tetrahydrochloride. Sections were counterstained with Harris's hematoxylin before being dehydrated and cover slipped. Palatine tonsils were used as positive controls, and for negative controls, the primary antibody was omitted during immunohistochemistry staining.

The membranous or cytoplasmic immunoexpression of cytokeratin 10 and 13 in epithelial cells of actinic cheilitis or of invasion front of lip squamous cell carcinomas were analyzed in 10 microscopic fields (400× magnification). Cytokeratin expression was analyzed in the basal, spinous, granular, and corneal layers of stratified squamous epithelium covering of the lip and labial mucosa.

For the evaluation of nuclear Ki-67 expression by epithelial cells, 10 microscopic fields in dysplastic areas of actinic cheilitis or of invasion front of LSCC (400× magnification) were digitally captured using an Axiocam camera (Axiocam MR3; Zeiss, Jena, Germany) attached to a light microscope (Axioskop 2 Plus; Zeiss) and recorded by the Axiovision software program (Axiovision 4.7; Zeiss). The cell proliferation index was determined from the ratio of the number of epithelial cells positive for $\mathrm{Ki}-67$ by the total number of epithelial cells in 10 consecutive microscopic fields in the dysplastic areas of actinic cheilitis and in invasion front of LSCC.

\section{Statistical analysis}

The association between the expression of CK10, CK13, and $\mathrm{Ki}-67$ in actinic cheilitis with and without epithelial dysplasia and lip squamous cell carcinomas was statistically compared using chi-square test. The correlation between the intensity of the dysplasia and the cell proliferation index was determined using the Spearman correlation coefficient. The level of significance for all statistical tests was set at $5 \%$. 


\section{Results}

In our sample, the actinic cheilitis (AC) and LSCC were frequently observed in men when compared with women (77.8 \%-AC and $60.0 \%$-LSCC). The age of patients with actinic cheilitis ranged from 33 to 82 years (average $=57.5$ ) and for LSCC ranged from 49 to 87 years (average $=68$ ). With regard to the risk factors, the majority of patients with actinic cheilitis $(53.3 \%)$ had a professional occupation related to prolonged exposure to the sunlight. For patients with lip squamous cell carcinoma, it was not possible to evaluate the occupation and the risk factors.

\section{Microscopic analyses of the actinic cheilitis and lip squamous cell carcinomas}

Microscopically, the hematoxylin-eosin stained sections of the 45 cases of actinic cheilitis revealed a hyperplastic vermilion in $84.5 \%$, the majority of them presenting orthokeratin $(64.5 \%)$. In some cases both parakeratin and orthokeratin were seen in the same section. Almost all cases of actinic cheilitis $(95.6 \%)$ showed some degree of basophilic alteration of collagen. According to the World Health Classification [3] for epithelial dysplasia, $26.6 \%$ of cases of actinic cheilitis did not present epithelial dysplasia and $73.4 \%$ of actinic cheilitis cases showed epithelial dysplasia $(24.4 \%$-mild dysplasia; $31.0 \%$-moderate dysplasia; $18.0 \%$-severe dysplasia) as illustrated in Fig. 1a-d.

The squamous cell carcinomas of the lip analyzed in this study were well-differentiated tumors (T1N0M0), microscopically characterized by intense keratinization, mild nuclear pleomorphism, several mitosis figures, and presence of moderate to intense mononuclear inflammatory infiltrate. The continuity of the tumor into the vermillion of the lip was constantly observed. In most tumors, the invasion pattern observed was composed of strands and islets of neoplastic cells.

\section{Immunoexpression of the cytokeratin 10 and 13 in actinic cheilitis and lip squamous cell carcinoma}

In the actinic cheilitis, CK13 was expressed in the spinous layer and granular layer of the vermilion and labial mucosa (Fig. 2a). In the basal layer, CK13 expression was weak or absent with loss of expression in the dysplastic areas of actinic cheilitis (Fig. 2c). Cytokeratin 10 was expressed in the spinous layer and granular layer of the vermilion (Fig. 2b). In the labial mucosa and in dysplastic areas of actinic cheilitis, CK10 expression was absent (Fig. 2d).
Fig. 1 Characteristic microscopic of actinic cheilitis. a Actinic cheilitis without epithelial dysplasia, with hyperorthokeratinized hyperplastic epithelium and basophilic change within the connective tissue. b Actinic cheilitis with mild dysplasia with abnormal epithelial architecture restricted to the lower third and the presence of a discrete cellular atypia. c Actinic cheilitis with moderate dysplasia with abnormal epithelial architecture extending to the middle third, the presence of moderate pleomorphism and hyperchromatic cell, and formation of pearl cornea. $\mathbf{d}$ Actinic cheilitis with severe dysplasia with abnormal epithelial architecture, loss of stratification, epithelial drop shaped, intense pleomorphism, and atypical mitosis (hematoxylin and eosin; 400×)
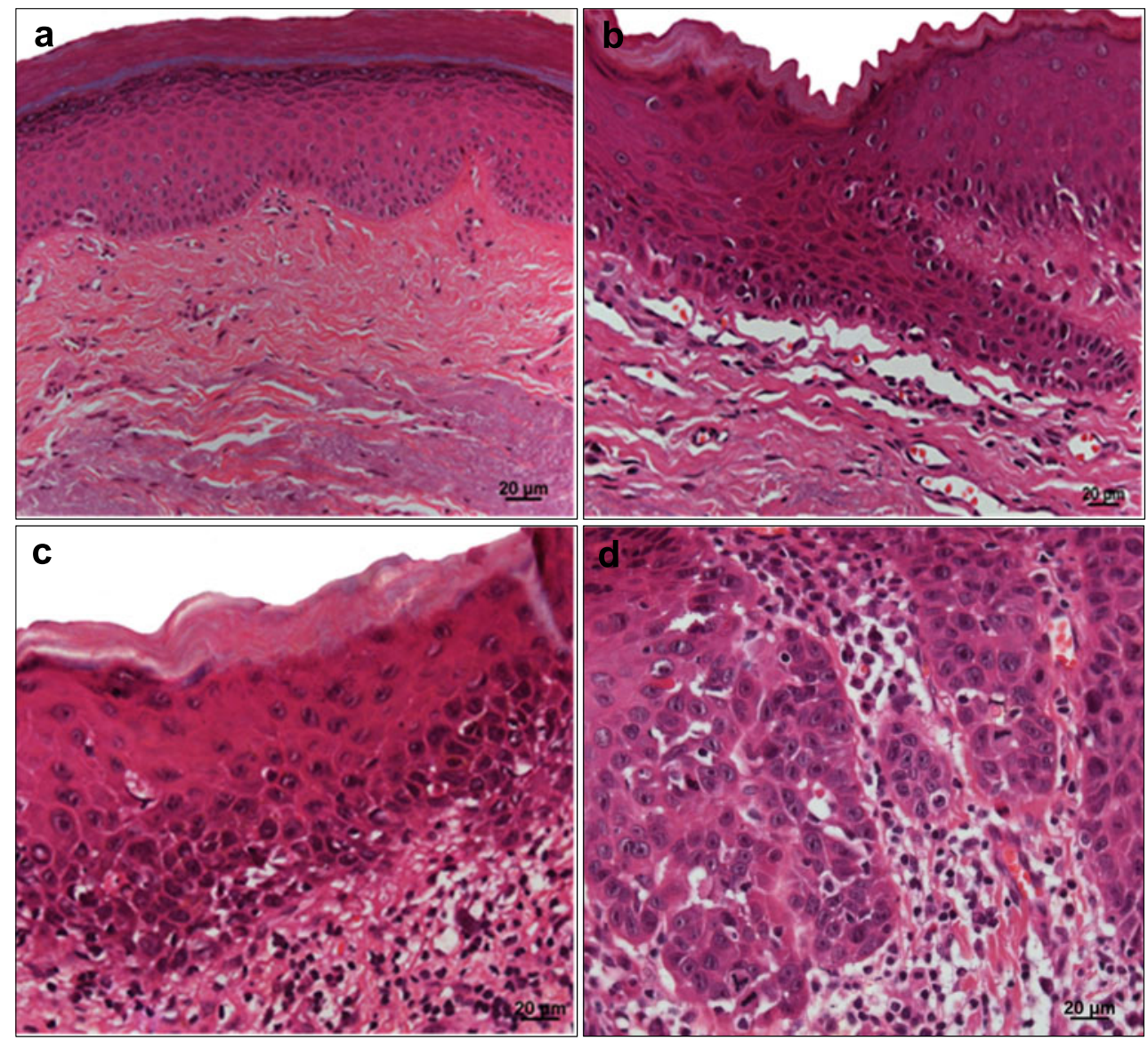
Fig. 2 CK13 and CK10 expression in actinic cheilitis with and without epithelial dysplasia and squamous cell carcinoma of the lip. a Actinic cheilitis without epithelial dysplasia, observe strong expression of cytokeratin 13 in all layers of the epithelium of the lip vermilion; $\mathbf{b}$ Actinic cheilitis without epithelial dysplasia, observe the expression of CK10 in granular and spinous layers of the epithelium and the absence of expression in the basal and suprabasal; c Actinic cheilitis with epithelial dysplasia, observe the heterogeneous expression of cytokeratin 13 in the spinous and granular layers of the epithelium. d Actinic cheilitis with epithelial dysplasia note the absence of CK10 expression in dysplastic regions. e SSC of the lip, note the heterogeneous expression of cytokeratin 13 in tumors lets and cords, with loss of expression in pearls corneas. f SSC of the lip, note the total absence of CK10 expression.

(immunohistochemistry: antiCK13 and anti-CK10; 200×)
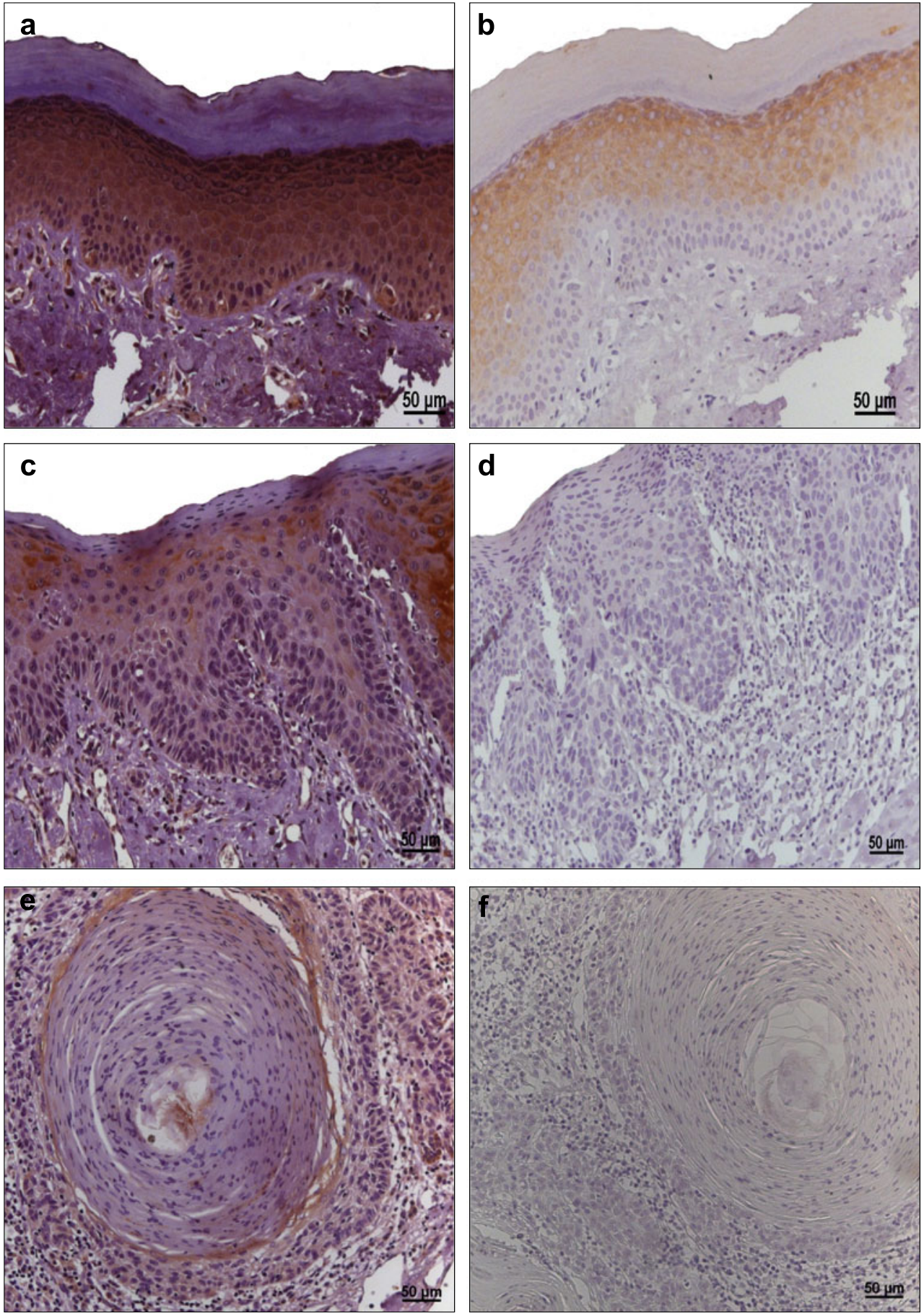

In lip squamous cell carcinoma, the expression of CK13 was observed mainly in the spinous layer of the vermilion border of the lip and the labial mucosa. The expression was focal and shown to be absent in peripheral cells and in keratin pearls when present (Fig. 2e). As regards CK10, positive markers were observed in the spinous layer, and in some specimens, they extended to the granular layer. However, in the epithelium of the labial mucosa and squamous cell carcinoma of the lip, there was no expression of CK10 as illustrated in Fig. 2 f.

There were no statistically significant differences between the expressions of cytokeratin CK13 and CK10 in the actinic cheilitis with and without epithelial dysplasia (Table 1). In addition, the expression of CK13 was similar and without statistical significance in LSCC and actinic cheilitis, including those with epithelial dysplasia as illustrated in Table 1. 
Table 1 Association between the expression of cytokeratin 13 (CK13) and 10 (CK10) in actinic cheilitis with and without epithelial dysplasia and lip squamous cell carcinoma

\begin{tabular}{|c|c|c|c|c|c|c|c|c|c|}
\hline \multirow[t]{3}{*}{ CKs } & \multirow{2}{*}{\multicolumn{2}{|c|}{$\frac{\mathrm{Ac}}{(n=12)}$}} & \multirow{2}{*}{\multicolumn{2}{|c|}{$\frac{\mathrm{ACD}}{(n=33)}$}} & \multirow{2}{*}{\multicolumn{2}{|c|}{$\frac{\mathrm{LSCC}}{(n=20)}$}} & \multirow{3}{*}{$\begin{array}{l}\mathrm{AC} \\
\times \\
\mathrm{ACD}\end{array}$} & \multirow{3}{*}{$\begin{array}{l}\text { ACD } \\
\times \\
\text { LSCC }\end{array}$} & \multirow{3}{*}{$\begin{array}{l}\text { AC } \\
\times \\
\text { LSCC }\end{array}$} \\
\hline & & & & & & & & & \\
\hline & $(+)$ & $(-)$ & $(+)$ & $(-)$ & $(+)$ & $(-)$ & & & \\
\hline CK13 & 11 & 1 & 33 & 0 & 19 & 1 & $p=0.267$ & $p=0.377$ & $p=1.000$ \\
\hline CK10 & 12 & 0 & 25 & 8 & 0 & 20 & $p=0.087$ & $p<0.001^{*}$ & $p<0.001^{*}$ \\
\hline
\end{tabular}

$C K$ cytokeratin, $C K 13$ cytokeratin13, $C K 10$ cytokeratin10, $A C$ actinic cheilitis without dysplasia, $A C D$ actinic cheilitis with dysplasia, $L S C C$ lip squamous cell carcinoma, $n$ number of specimens analyzed, $p$ descriptive level of chi-square

${ }^{*} p<0.001$ statistically significant

In contrast, we detected a statistically significant difference between the CK10 expression in LSCC in comparison with actinic cheilitis with epithelial dysplasia $(p<0.001)$ and without epithelial dysplasia $(p<0.001)$ (Table 1). All actinic cheilitis without epithelial dysplasia and $76 \%$ of those with epithelial dysplasia were positive for CK10; however, the malignant cells of LSCC did not express cytokeratin 10.

\section{Proliferation index obtained by Ki-67 expression}

The proliferative activity, visualized by Ki-67 staining, was observed mainly in the dysplastic areas of actinic cheilitis and in invasion front of LSCC. Based on the median values of actinic cheilitis without dysplasia, the sample was divided into two groups: $\leq 25 \%$ of epithelial cells positive for Ki- 67 and $>25 \%$ of epithelial cells positive for Ki-67 (high cell proliferation index).

In Table 2, the distribution of actinic cheilitis with and without epithelial dysplasia may be observed, and of lip squamous cell carcinoma based on the cell proliferation index, determined by Ki-67 expression. The actinic cheilitis with epithelial dysplasia and LSSC showed a high proliferative rate ( $\geq 25 \%$ of epithelial cells positive for Ki-67) as demonstrated

Table 2 Distribution of actinic cheilitis with and without and LSCC to cell proliferation index determined by Ki-67 expression

\begin{tabular}{llll}
\hline & AC & ACD & LSCC \\
Ki-67 & $N(\%)$ & $N(\%)$ & $N(\%)$ \\
\hline$<25 \%$ & $10(83.4 \%)$ & $5(17.9 \%)$ & $6(30.0 \%)$ \\
$\geq 25 \%$ & $2(16.6 \%)$ & $28(82.1)$ & $14(70.0 \%)$ \\
Total & $12(100 \%)$ & $33(100 \%)$ & $20(100 \%)$
\end{tabular}

$A C$ actinic cheilitis without dysplasia, $A C D$ actinic cheilitis with dysplasia, $L S C C$ lip squamous cell carcinoma, $p$ descriptive level of chi-square test

${ }^{*} p<0.001$ statistically significant in Table 2. On the other hand, $83.4 \%$ of the actinic cheilitis without epithelial dysplasia presented a low proliferation index $(<25 \%$ epithelial cells positive for Ki-67). There was a statistically significant difference $(p<0.001)$ between the high and low rates of cell proliferation in actinic cheilitis with and without dysplasia and LSCC.

To find out whether there was correlation between the proliferation index determined by Ki-67 and the degree of epithelial dysplasia, Spearman's test was performed. Our results proved that there was a positive and significant correlation between $(r=0.074$ and $p<0.001)$ the level of epithelial cell proliferation, determined by $\mathrm{Ki}-67$, and the degree of epithelial dysplasia in actinic cheilitis. In Fig. 3, it may be seen that the degree of epithelial dysplasia is directly proportional to increase of cell proliferation index.

\section{Discussion}

In the majority of cases of actinic cheilitis, a clinical presentation does not necessarily correlate with the degree of histologic abnormality. Therefore, in some institutions, vermillionectomy has been the treatment of choice for actinic cheilitis, since this approach allows a histopathological evaluation of all lip tissue removed, including the possibility of analyzing, when present, the invasion front in squamous cell carcinoma [7, 8]. Based on this collection of information, in the present study, we analyzed 65 surgical specimens obtained from vemillionectomy of patients with actinic cheilitis.

The main objective in our study was to verify whether cytokeratin expressions are correlated with the degree of epithelial dysplasia. The discrepancy of epithelial dysplasia in the oral lesions has been the focus of many discussions among researchers $[1,2,4,5]$, and none of the currently available molecular markers have proved to be prognostically significant [4.5]. Actinic cheilitis is often associated with intense inflammatory infiltrate, which may make it difficult to establish the degree of epithelial dysplasia.

Although the expression of cytokeratin 10 and 13 has been widely investigated in oral malignant and precancerous lesions [10-18], only one of these studies was conducted in actinic cheilitis [10].

In the present study, the cytokeratin expressions were analyzed in the basal, spinous, granular, and corneal layers of the vermilion and labial mucosa.

The expression of CK13 was observed in the majority of actinic cheilitis with or without epithelial dysplasia (Table 1). Strong CK13 expression was detected mainly in the spinous and granular layer of the vermilion and labial mucosa; however, in the basal layer, a less intense expression or even absence of expression was observed (Fig. 2a). In dysplastic areas of actinic cheilitis, there was loss of expression (Fig. 2c). Thus, CK13 expression varied according to the degree of 
Fig. 3 Distribution of actinic cheilitis without and with different degrees of dysplasia according to the index of proliferation determined by $\mathrm{Ki}-67$ expression. Spearman's test, $r=$ 0.074

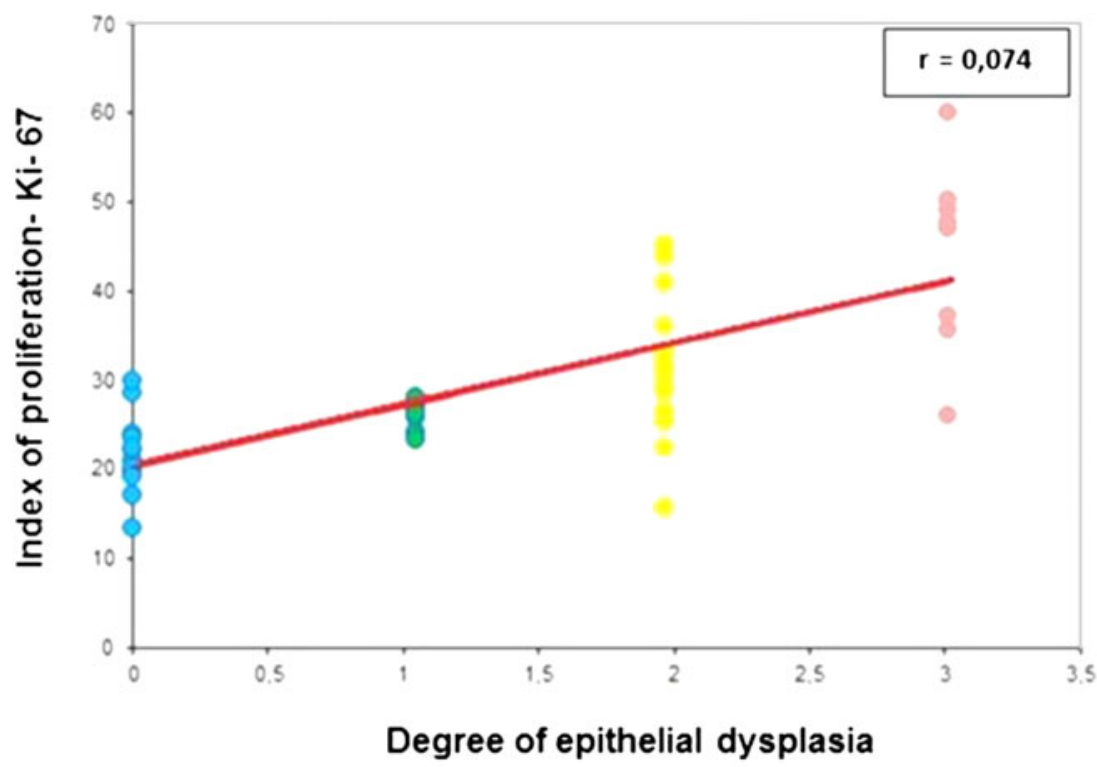

0 - Without epithelial dysplasia
1 - Mild epithelial dysplasia
2 - Moderate epithelial dysplasia
3 - Severe epithelial dysplasia epithelial dysplasia, the more intense the epithelial dysplasia was, the fewer the epithelial areas that retained the expression. In lip squamous cell carcinoma, the expression of CK13 was focal and also shown to be absent in peripheral cells and in keratin pearls when it was present (Fig. 2e).

These results reinforce previous findings, which confirmed that the loss of CK13 expression in dysplastic epithelium appears to follow the process of malignant transformation [11, 13, 15-17].

In the same way as observed by Kitamura et al. [17] in oral leukoplakia and oral squamous cell carcinomas, we also observed that expression of CK13 is strongly expressed in the epithelium of the labial mucosa and consistently decreases in dysplastic areas and invasive squamous cell carcinomas Furthermore, heterogeneous positivity of CK13, as was found in our LSCC, is in agreement with the results of Gires et al. [11] who analyzed the expression of CK13 in leukoplakia and oral squamous cell carcinoma and concluded that the downregulation of CK13 expression in dysplastic areas and in LSCC is directly correlated with the intensity of the epithelial dysplasia. Thus, this biomarker is effective in the distinction between an abnormal epithelium and a dysplastic epithelium.

Contrasting with our results, Santos et al. [10] showed that CK13 is expressed in the suprabasal layers of the epithelium of the lip, but they failed to link the decreased of this expression with epithelial dysplastic changes in actinic cheilitis. However, these authors admitted that focal cellular distribution was observed, which may indicate that in dysplastic conditions, the process of differentiation of the epithelium occurs.
The cytokeratin 10 had a similar behavior to CK13, and it was expressed in the spinous layer and granular layer of the vermilion (Fig. 2b). In labial mucosa, in dysplastic areas of actinic cheilitis and LSCC, the CK10 expression was absent (Fig. 2d, f). These results reinforce those found by Fillies et al. [12] who suggested that the absence of expression of CK10 is associated with the presence of epithelial dysplasia in potentially malignant lesions such as oral leukoplakia.

Our results also confirm some findings of Santos et al. [10], who verified the expression of CK10 expressed in the spinous layer and granular layer of the vermilion in most cases of actinic cheilitis. In spite of these authors having concluded that that the expression of CK10 was independent of the degree of epithelial dysplasia, they claimed that the presence of CK10 in the intermediate layers of the epithelium supports the thought that an altered keratinization process is taking place.

In the present study, there was a statistically significant association between the expression of CK10 in LSCC and actinic cheilitis with epithelial dysplasia $(p<0.001)$ or without epithelial dysplasia $(p<0.001)$. This confirms the expression of CK10 as a biomarker of the process of malignant transformation of epithelial cells because its expression is being reduced with increasing structural and cytological changes observed in dysplastic areas and is totally negative in squamous cell carcinomas of the lip.

In order to compare the expression of cytokeratins in actinic cheilitis and LSSC with the cell proliferation index, we used Ki-67 immunostaining, whereas the sample was divided into two groups: $\leq 25 \%$ of positive epithelial cells and $>25 \%$ of positive epithelial cells (high cell proliferation index). In the 
group of actinic cheilitis with epithelial dysplasia and LSSC, the majority showed a high proliferative rate $(\geq 25 \%$ of epithelial cells positive for Ki-67) (Table 2). Therefore, there was a statistically significant difference $(p<0.001)$ between the high and low rates of cell proliferation in cheilitis with and without dysplasia and LSCC.

Some previous studies have reported the increased number of proliferating epithelial cells in epithelial dysplasia and squamous cell carcinoma, determined by means of Ki-67 immunostaining, also suggesting that the increase in the population of proliferating cells in the suprabasal layer of the oral epithelium is a useful marker for identifying high degrees of dysplasia [19-22].

In the present study, to find out whether there was a correlation between the proliferation index determined by Ki-67 and the degree of epithelial dysplasia, Spearman's test was performed and the value found was $r=0.074$ and $p<0.001$. These results suggest that the level of proliferation determined by Ki-67 is directly correlated with the intensity of the epithelial dysplasia, confirming previous studies [19-22].

Altogether, our results suggest that the CK13 and CK10 participate of malignant transformation of epithelial cells in actinic cheilitis, and these cytokeratins should be used as predictive biomarkers of epithelial dysplastic areas or invasive squamous cell carcinoma in the lip region.

\section{Conclusion and clinical relevance}

This study is the first to examine the expression of CK10 and CK13 in association with the cell proliferation index, which was determined by Ki-67 immunostaining, in actinic cheilitis with and without epithelial dysplasia and squamous cell carcinomas of the lip. These results provide evidence that there is a downregulation of CK10 expression in dysplastic areas of patients with actinic cheilitis and in those with LSCC and that the index of cell proliferation, determined by $\mathrm{Ki}-67$, is directly correlated with the intensity of the epithelial dysplasia. Altogether, these results suggest that CK10 expression and the epithelial cell proliferation index can help to identify malignant transformation in the lip region.

Acknowledgments The authors wish to thank the CAPES (Coordenação de Aperfeiçoamento de Pessoal de Nível Superior) and $\mathrm{CNPq}\left(\mathrm{N}^{\circ}: 141641 / 2013-4\right)$ for supporting this study.

Conflict of interest The authors declare that they have no competing interests.

\section{References}

1. Bouquot J, Speight PM, Farthing PM (2006) Epithelial dysplasia of the oral mucosa - diagnostic problems and prognostic features. Current Diagnostic Pathology 12:11-12

2. Speight PM (2010). An overview of the classification and predictive value of oral epithelial dysplasia [http://www.iaop.com/docs/ epithelial\%20dysplasia.pdf] Accessed 26 June 2012.

3. Gale N, Pilch BZ, Sidransky D, Westra WH, Califano J (2005) Epithelial precursor lesions. In: Barnes L, Eveson JW, Reichart P, Sidransky D (eds) World Health Organization Classification of Tumours. Pathology and genetics: head and neck tumours. IARC Press, Lyon, pp 140-142

4. Kujan O, Khattab A, Oliver RJ, Roberts SA, Thakker N, Sloan P (2007) Why oral histopathology suffers inter-observer variability on grading oral epithelial dysplasia: an attempt to understand the sources of variation. Oral Oncol 43(3):224-31

5. Warnakulasuriya S, Reibel J, Bouquot J, Dabelsteen E (2008) Oral epithelial dysplasia classification systems: predictive value, utility, weaknesses and scope for improvement. J Oral Pathol Med 37(3): $127-33$

6. Wood NH, Khammissa R, Meyerov R, Lemmer J, Feller L (2011) Actinic cheilits: a case report and review of the literature. Eur J Dent 5(1):101-6

7. Jadotte YT, Schwartz RA (2012) Solar cheilosis: an ominous precursor: part I. Diagnostic insights. J Am Acad Dermatol 66(2):173-84

8. Vieira RA, Minicucci EM, Marques ME, Marques AS (2012) Actinic cheilitis and squamous cell carcinoma of the lip: clinical, histopathological and immunogenetic aspects. An Bras Dermatol 87(1):105-14

9. Menta Simonsen Nico M, Rivitti EA, Lourenço SV (2007) Actinic cheilitis: histologic study of the entire vermilion and comparison with previous biopsy. J Cutan Pathol 34(4):309-14

10. dos Santos JN, de Sousa SO, Nunes FD, Sotto MN, de Araújo VC (2003) Altered cytokeratin expression in actinic cheilitis. J Cutan Pathol 30(4):237-41

11. Gires O, Mack B, Rauch J, Matthias C (2006) CK8 correlates with malignancy in leukoplakia and carcinomas of the head and neck. Biochem Biophys Res Commun 343(1):252-9

12. Fillies T, Jogschies M, Kleinheinz J, Brandt B, Joos U, Buerger H (2007) Cytokeratin alteration in oral leukoplakia and oral squamous cell carcinoma. Oncol Rep 18(3):639-43

13. Ohta K, Ogawa I, Ono S, Taki M, Mizuta K, Miyauchi M, Takechi M, Shigeishi H, Takata T, Kamata N (2010) Histopathological evaluation including cytokeratin 13 and $\mathrm{Ki}-67$ in the border between Lugol-stained and -unstained areas. Oncol Rep 24(1):9-14

14. Schaaij-Visser TB, Bremmer JF, Braakhuis BJ, Heck AJ, Slijper M, van der Waal I, Brakenhoff RH (2010) Evaluation of cornulin, keratin 4, keratin 13 expression and grade of dysplasia for predicting malignant progression of oral leukoplakia. Oral Oncol 46(2):123-7

15. Sakamoto K, Aragaki T, Morita K, Kawachi H, Kayamori K, Nakanishi S, Omura K, Miki Y, Okada N, Katsube K, Takizawa T, Yamaguchi A (2011) Down-regulation of keratin 4 and keratin 13 expression in oral squamous cell carcinoma and epithelial dysplasia: a clue for histopathogenesis. Histopathology 58(4):531-42

16. Ida-Yonemochi H, Maruyama S, Kobayashi T, Yamazaki M, Cheng J, Saku T (2012) Loss of keratin 13 in oral carcinoma in situ: a comparative study of protein and gene expression levels using paraffin sections. Mod Pathol 25(6):784-94

17. Kitamura R, Toyoshima T, Tanaka H, Kawano S, Kiyosue T, Matsubara R, Goto Y, Hirano M, Oobu K, Nakamura S (2012) Association of cytokeratin 17 expression with differentiation in oral squamous cell carcinoma. J Cancer Res Clin Oncol 138(8):1299-310 
18. Kobayashi T, Maruyama S, Abé T, Cheng J, Takagi R, Saito C, Saku T (2012) Keratin 10-positive orthokeratotic dysplasia: a new leucoplakia-type precancerous entity of the oral mucosa. Histopathology 61(5):910-20

19. Gonzalez-Moles MA, Ruiz-Avila I, Rodriguez-Archilla A, Martinez-Lara I (2000) Suprabasal expression of Ki-67 antigen as a marker for the presence and severity of oral epithelial dysplasia. Head Neck 22(7):658-61

20. Takeda T, Sugihara K, Hirayama Y, Hirano M, Tanuma JI, Semba I (2006) Immunohistological evaluation of Ki-67, p63, CK19 and p53 expression in oral epithelial dysplasias. J Oral Pathol Med 35(6):369-75

21. González-Moles MA, Bravo M, Ruiz-Avila I, Acebal F, GilMontoya JA, Brener S, Esteban F (2010) Ki-67 expression in non-tumour epithelium adjacent to oral cancer as risk marker for multiple oral tumours. Oral Dis 16(1):68-75

22. Montebugnoli L, Gissi DB, Badiali G, Marchetti C, Cervellati F, Farnedi A, Foschini MP (2011) Ki-67 from clinically and histologically "normal" distant mucosa as prognostic marker in early-stage (T1-T2N0) oral squamous cell carcinoma: a prospective study. J Oral Maxillofac Surg 69(10):2579-84 